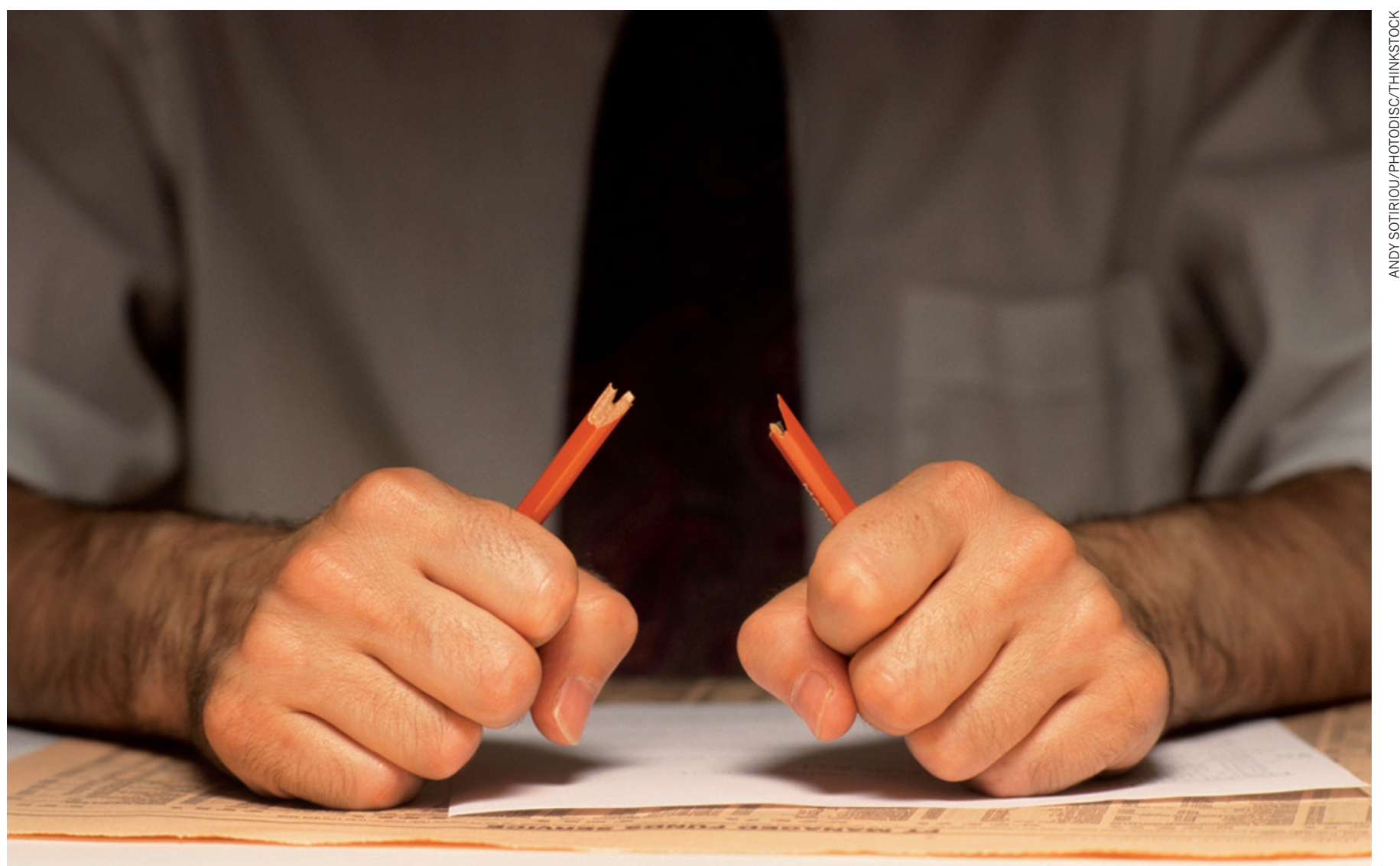

Beyond raising a person's blood pressure, stress can increase the odds of blood clots forming, leading to heart attacks and stroke.

\title{
PSYCHOLOGY
}

\section{Mind over myocardium}

\section{Mental factors beyond stress trigger physiological changes that can cause heart disease.}

\section{BY SHAILAJA NEELAKANTAN}

A middle-aged man has a stressful job and deals with depression. One day he felt his chest tighten, a sensation that gradually turned to pain. An angiogram showed his arteries free of blockage. Yet he soon suffered a heart attack.

"We see this commonly. Individuals have classic signs like chest pains, and we don't find any artery blockages," says Arun Kumar, a cardiologist at the University of Missouri School of Medicine in Columbia. "These symptoms can be a response to emotional stress and anxiety and not mediated through the common mechanism of coronary heart disease, which is atherosclerosis," says Kumar. "This just goes to show how important the mind is and how it influences the body." For instance, a 2010 study that ranked 291 diseases for the years of disability they cause around the world ranked major depressive disorder eleventh, a rise from fifteenth in 1990 (ref. 1). This suggests that depression is taking an increasing toll on people's lives.

In the past couple of decades, psychological factors such as stress and depression have become more recognized as factors that affect cardiac health ${ }^{2}$. And although doctors have been advising heart-disease patients to avoid stress, psychological factors continue to trigger heart attacks - even in individuals who show no signs of cardiovascular impairment, such as blocked arteries. In some cases, depression increases the odds of an artery blockage further from the heart, which also increases the odds of having a heart attack ${ }^{3}$.

\section{NERVE-WRACKED RHYTHM}

It now appears that other disturbances in our state of mind can also afflict the heart. Hostility, job stress, anxiety and depression can all lead to raised pulse and steroid levels, elevated white-blood-cell counts and poor lifestyle choices. And despite the subjective nature of psychological influences on physical health,

many clinicians and scientists acknowledge the mounting data and research that link these factors to heart disease. One study even showed a small decrease in cardiac-related deaths when people with heart disease are provided with psychological therapy, but it did not demonstrate that this approach reduced non-fatal heart attacks ${ }^{4}$.

"Stress increases adrenaline and steroid levels, which increases pulse rate and blood pressure and creates clotting tendencies," says cardiologist K. Srinath Reddy, president of the Public Health Foundation of India in New Delhi. Reddy says that pulse rate, long disregarded as a valuable indicator of heart disease, is now seen as a strong predictor of future chronic heart disease. "The thinking

DNATURE.COM Stress makes its molecular mark through epigenetics: go.nature.com/oaxqt5 has changed though in the last 10 to 15 years," Reddy says. The emphasis on the role of high blood pressure and cholesterol overlooked an 
important connection. While conceding that those factors are important, Reddy says they are in fact more easily measured manifestations of a root cause.

Similarly, clinical depression causes physical changes that could lead to cardiovascular disease. The body of someone suffering from depression is in a constant state of stress, explains Maen Nusair, a cardiologist at the University of Missouri School of Medicine in Columbia. This stress, Nusair says, generates hyperactivity in the brain's hypothalamicpituitary axis, and blood becomes sticky and liable to form clots - the most common underlying mechanism of heart disease.

In addition, people who suffer regular bouts of depression have a higher white-blood-cell count - a sign of inflammation - that might 崖 lead to coronary heart disease, says Hester Duivis, a doctoral student who is studying the role of psychology in somatic diseases at Tilburg University in the Netherlands ${ }^{5}$. "Inflammation is involved in the thickening of arteries [from] which small clots can come loose and end up in the heart, so arteries are blocked and one gets a heart attack," says Duivis.

Similar to emotional stress, increased steroid and adrenaline levels constrict blood vessels, raise blood pressure and damage the lining of blood vessels, which can cause angina (chest pain caused by a lack of blood flow or oxygen to the heart).

"Stress increases adrenaline and steroid levels, which increases pulse rate and blood pressure and creates clots." "Adrenaline cracks open the plaque, and this leads to chronic damage of blood vessels," explains Reddy. Moreover, she says, adrenaline also causes electrical disturbances to the heart. Although earlier thinking went that heart attacks were caused by blocked blood vessels, evidence now suggests that even a plaque rupturing can generate a blood clot that leads to a heart attack, Reddy adds.

Research by Gregory Harshfield, a cardiologist at Georgia Health Sciences University in Augusta, shows that stress can cause people to retain more sodium in their bodies than was previously thought. "Some patients retain sodium equivalent to a small bag of fries during stress," says Harshfield, with an accompanying — and potentially fatal — rise in blood pressure.

\section{TYPE A RIDES AGAIN}

In 1959, cardiologists Meyer Friedman and Ray Rosenman proposed that a type A personality - behaviour characterized by excessive competitiveness, irritability, hostility, a persistent desire for recognition, and so on - could double the risk of coronary heart disease in otherwise healthy individuals. It was subsequently suggested that only a few type A traits, such as hostility, were linked to heart disease. Nonetheless, recent research supports a link between

\section{RISING STRESS IN THE USA}

Nearly half of US adults say that their stress levels increased over the past five years.

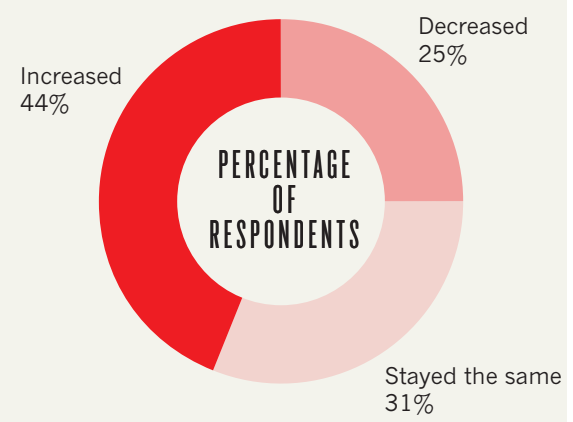

various type A behaviours and heart disease.

"There is too much emphasis on depression," says Giovanni Fava, a clinical psychologist at the University of Bologna in Italy. "Type A behaviours are common psychological constructs and must be recognized for their possible relation to heart disease." Fava says that much research linking type A traits to heart health has been flawed because of overreliance on self-administered questionnaires. To get more reliable data, Fava and colleagues interviewed 1,398 patients. They concluded that type A people were disproportionately represented in a population of people with cardiovascular disease: type A's make up $36.1 \%$ of cardiac patients, but only $10.8 \%$ of non-cardiac patients were type A (ref. 6).

Fava points to other findings that support the type A link to heart health. "If we have patients with major depression and they are randomized with psychotherapy or drug treatment and if treatment is working, you would expect they would do better in cardiological parameters," he says. But evidence suggests that this is not the case. Moreover, he says, a type A person who gets depressed and has a heart attack will often revert to risky type A behaviours even if their depression is treated. This, he says, makes a compelling case for the necessity of treating type A traits directly, as they are the source from which so many cardiovascular troubles flow.

\section{VICIOUS CIRCLE}

Such focus is especially important because stress in a type A person, even more so than in most people, becomes self-reinforcing. Such people often make their lives more stressful by, for example, committing to work beyond their capacity. But long, intense working days can cause depression, which, in turn, leads to more stress and raises the possibility of heart problems, says Marianna Virtanen, an epidemiologist at the Finnish Institute of Occupational Health in Helsinki. Virtanen and her colleagues reported that long working days (which they defined as working 11 hours or more a day) and job strain create about a $40 \%$ excess risk of coronary heart disease ${ }^{7}$.

"Pushy, aggressive, hostile and angry people have bad stress and are predisposed to high blood pressure and arrhythmia, and therefore are at increased risk of myocardial disease," says Alok Bajpai, a psychiatrist at the Indian Institute of Technology, Kanpur. And beyond direct physiological impacts, psychological challenges often contribute to poor lifestyle choices, such as alcohol and smoking addictions, poor diet and lack of exercise - all factors that themselves put individuals at greater risk of heart disease.

\section{BENEFITS OF BALANCE}

Still, not all stress is bad. Reddy points out that stress is instinctive. "Hunter-gatherers had to flee or fight every day," he says. That would have produced the same reactions, such as increased adrenaline, heart rate and steroid levels, but they were exercising when hunting and gathering. That was protective stress. Today, says Reddy, "the muscles are not exercising to counterbalance that and there is no outlet for stress."

The key to the mind and heart revolves around balance - keeping stress and other psychological factors in check, and refraining from bad lifestyle choices. Bajpai points out that stress, depression and type A behaviours are becoming more prevalent in emerging economies such as India and China. As a result, he says, those populations are going to experience a doubling in the incidence of heart attacks and a tripling in the numbers of heart attack-related deaths. And these countries are not starting from a healthy baseline: some studies indicate that more than one in three Indians already suffers from depression ${ }^{8}$.

Many healthcare professionals often assume that growing affluence leads to a richer diet, which increase cardiovascular disease. Bajpai notes, however, that it is not just diet but also the Western lifestyle of stress and corporate culture that can have as big an impact as poor eating at breakfast, lunch and dinner. The overall balance of mind and body offers some of the best medicine for cardiovascular health around the world.

Shailaja Neelakantan is a freelance science writer based in New Delhi.

1. Murray, C. J. L. et al. Lancet 380, 2197-2223 (2012).

2. Nusair, M., Al-dadah, A. \& Kumar, A. Mol. Med. 109, 199-203 (2012).

3. Grenon, S. M. et al. J. Am. Heart Asssoc. doi: 10.1161/JAHA.112.002667 (2012)

4. Whalley, B., Thompson, D. R. \& Taylor, R. S. Int. J. Behav. Med. doi: 10.1007/s12529-012-9282-x (2012)

5. Duivis, H. E. et al. Psychoneuroimmunology, advance online publication doi: 10.1016 j.psyneuen.2012.07.006 (18 August 2012)

6. Sirri, L. et al. Int. J. Clin. Pract. 66, 854-861 (2012).

7. Virtanen, M. et al. Am. J. Epidemiol. 176(7), 586-596 (2012); published online 5 September 2012.

8. Bromet, E. et al. BMC Med. 9, 90-105 (2011) 